Bull. Austral. Math. Soc.

$05 \mathrm{~B} 15,05 \mathrm{~B} 30,62 \mathrm{\kappa} 15$

VoL. 59 (1999) [351-351]

\title{
Orthogonal main effect plans and an extension to resolution $V$
}

\author{
LEONIE BARBara Burgess
}

Orthogonal main effect plans (OMEPs) are a special class of fractional factorial designs, in which all main effects can be orthogonally estimated, assuming that all interactions between factors are negligible. In this thesis we consider three algorithmic methods for obtaining OMEPs with different numbers of repeated runs. We also construct, by a fourth method, OMEPs of resolution $V$, designs which allow orthogonal estimation of the main effects as well as of all two-factor interactions, assuming that interactions between three or more factors are negligible.

Chapter 1 gives the preliminary definitions and previous results in the area. Chapter 2 discusses two algorithmic techniques, a backtrack algorithm and solving a system of equations, for obtaining orthogonal main effect plans. A third method, an interchange algorithm for three factor OMEPs, is given in Chapter 3, followed by a comparison of all three methods in Chapter 4. A construction for four factor OMEPs is given in Chapter 5 and Chapter 6 discusses orthogonal main effect plans of resolution $V$.

School of Mathematical Sciences

University of Technology, Sydney

PO Box 123

Broadway, NSW 2007

Australia

Received 16th November, 1998

Thesis submitted to the University of New South Wales, August 1997. Degree approved, July 1998. Supervisor: Associate Professor Deborah J. Street.

Copyright Clearance Centre, Inc. Serial-fee code: 0004-9729/99 $\$ A 2.00+0.00$. 